\title{
Privatsphäre mit System
}

\section{Symposium der LDI NRW}

Wie lässt sich die Privatsphäre in vernetzten IT-Systemen schützen? Wie passen ITGrundrecht und informationelle Selbstbestimmung zusammen mit Cloud Computing und Behavioral Targeting? Diese Fragen waren das Thema des Symposiums „Privatsphäre mit System - Datenschutz in einer vernetzten Welt", das die Landesbeauftragte für Datenschutz und Informationsfreiheit Nordrhein-Westfalen gemeinsam mit dem Institut für Informations-, Telekommunikations- und Medienrecht der Universität Münster im November 2009 in Düsseldorf veranstaltete.

Das Bundesverfassungsgericht hat mit dem Grundrecht auf Gewährleistung der Vertraulichkeit und Integrität informationstechnischer Systeme einen Ansatz entwickelt, der ein wichtiger Anstoß für die Rechtsentwicklung sein kann. Die technische Entwicklung bringt erhebliche Risiken für die Privatsphäre mit sich. Die Vorträge befassten sich deshalb zunächst mit dem ITGrundrecht und den internationalen Instrumenten zum Schutz der Privatsphäre. Danach wurden beispielhaft zwei Konzepte untersucht, bei denen Gefährdungen der Privatsphäre besonders nahe liegen: Cloud Computing und Behavioral Targeting.

Zur Eröffnung des Symposiums wies Roland Schlapka ${ }^{1}$ darauf hin, dass Datenschutz weiterhin „Konjunktur“ habe. Nach 30 Jahren Datenschutz in Nordrhein-Westfalen werde das Thema auch außerhalb der Fachwelt wieder als wichtig eingeschätzt. Gerade vernetzte Systeme werfen die Frage auf, wie der Schutz der informationellen Selbstbestimmung sowie der Vertraulichkeit und Integrität sicherzustellen ist.

Prof. Dr. Matthias Bäcker ${ }^{2}$ erläuterte Funktion und Schutzbereich des ITGrundrechts. Er wies darauf hin, dass der Schutzbereich noch zu konturieren sei: So werde zu diskutieren sein, wann ein System hinreichend komplex ist, um in den Schutzbereich zu fallen. Es frage sich auch, inwiefern ein System einer Person zugeordnet sein muss und wie sich die Rechte mehrerer Grundrechtsträger zueinander verhalten. Zur Abgrenzung zum Fernmeldege-

1 Ständiger Vertreter der Landesbeauftragten für Datenschutz und Informationsfreiheit Nordrhein-Westfalen

2 Juniorprofessur für Öffentliches Recht, Universität Mannheim heimnis stellte er die Rechtsprechung der Bundesverfassungsgerichtssenate dar. Eine mögliche Entwicklung sei der Ausbau des IT-Grundrechts zum Recht auf Schutz digitaler Privatheit. Im Verhältnis zum Recht auf informationelle Selbstbestimmung sei das IT-Grundrecht spezieller. Auswirkungen der Gewährleistungsdimension seien etwa im Arbeitsrecht, im IT-Vertragsrecht oder im Deliktsrecht denkbar.

Thomas Zerdick ${ }^{3}$ gab einen Überblick über internationale und supranationale Regelungen zum Datenschutz, die Entwicklungen in der EU und Ansätze für internationale Standards. Er machte deutlich, dass es noch viele weiße Flecken ohne Datenschutzrecht auf der Weltkarte gibt. Einige internationale oder supranationale Rechtstexte regelten aber auch Datenschutzfragen, wie etwa im Rahmen der Vereinten Nationen, der OECD oder der APEC. In der EU seien die Datenschutzregelungen harmonisiert. Das Recht auf Schutz der personenbezogenen Daten werde auch mit der EU-Grundrechtecharta festgeschrieben. In verschiedenen Zusammenhängen werden gemeinsame Standards angestrebt, so etwa durch den Europarat oder in der Madrider Erklärung der internationalen Datenschutzkonferenz. Schließlich plane die EU-Kommission 2010 die Evaluierung des europäischen $\mathrm{Da}$ tenschutzrechts, in die auch die Ergebnisse der laufenden öffentlichen Anhörung einbezogen werden.

Prof. Dr. Dogan Kesdogan ${ }^{4}$ stellte aus technischer Sicht dar, wie sich Einzelsysteme und verteilte Systeme unterscheiden. Während bei Einzelsystemen Sicherheit ausgehend von der Hardware aufgebaut werde, werde in public Clouds von der Anwendungssoftware her gedacht. Dies setze zunächst „blindes“ Vertrauen in den Softwareanbieter voraus. Transparenz und Auditierungen bieten allenfalls einen gewissen Ausgleich. Abgegrenzte Anwendungsbereiche wie die Speicherung gut verschlüsselter Daten können auch in verteilten Systemen sicher gestaltet werden. Für die sichere Datenbearbeitung in public Clouds stehen technisch sichere Lösungen aber noch aus.

3 EU-Kommission, General-Direktion Justiz, Freiheit und Sicherheit, Referat Datenschutz

4 Leiter des Lehrbereichs für IT-Sicherheit im Institut für Wirtschaftsinformatik an der Universität Siegen
Dr. Armgard von Reden ${ }^{5}$ betrachtete Cloud Computing aus rechtlicher Sicht. Verteilte Systeme seien jedenfalls als private Clouds in bestimmten Konstellationen, bei sorgfältig gestalteten Bedingungen und in einem abgegrenzten Rechtsrahmen rechtskonform denkbar. So könne eine Cloud für nicht-sensible Daten innerhalb Europas möglich sein. Sensible Daten können dabei wohl nur innerhalb nationaler Grenzen verarbeitet werden. Es komme immer auch darauf an, die Verantwortung gesetzlich und vertragsrechtlich sicherzustellen.

Zur verhaltensorientierten Zielgruppenansprache für die Werbung im Internet machte Dr. Thilo Weichert ${ }^{6}$ deutlich, unter welchen Umständen Nutzungsanalysen für Werbezwecke datenschutzkonform möglich sind. Nach dem TMG sei nur eine pseudonyme Analyse zulässig. Auf die Möglichkeit eines Widerspruchs müsse vor der Erfassung hingewiesen werden. Sensible Kategorien und personenbeziehbare Klickhistorien müssen vermieden werden. Vorbildliche Anbieter des Behavioral Targeting schalteten einen Anonymisierer ein und trennten die Inhaltsdaten von den Identitätsdaten. Die Analyse von Kommunikationsinhalten etwa durch E-Mail-Dienste sei nach deutschem Recht unzulässig. Der am häufigsten eingesetzte Analysedienst eines US-amerikanischen Anbieters sei mit deutschem Recht nicht vereinbar.

Thema der Abschlussdiskussion, die Prof. Dr. Bernd Holznagel ${ }^{7}$ moderierte, war die Frage, ob das vorhandene Datenschutzrecht die Anforderungen durch die weiterentwickelte Technik und den häufig internationalen Anwendungsbedarf erfüllen kann. Es zeigte sich, dass sorgfältig geprüft werden muss, ob ein neuer Systemdatenschutz oder ein neues Datenschutzsystem erforderlich ist.

Der Tagungsband zum Symposium wird voraussichtlich im Februar 2010 erscheinen (www.ldi.nrw.de).

Nils Schröder, Landesbeauftragte für $\mathrm{Da}$ tenschutz und Informationsfreiheit NRW, Referat Öffentlichkeitsarbeit

5 Chief Privacy Officer der IBM Europe, Middle East \& Africa

6 Leiter des Unabhängigen Landeszentrums für Datenschutz Schleswig-Holstein

7 Institut für Informations-, Telekommunikations- und Medienrecht, Westfälische Wilhems-Universität Münster 